\title{
COVID-19 Clusters at Workplaces and its Transmission into Communities in Vietnam: A Novel Emerging Occupational Risk Factor at Work Due to Coronavirus Infection
}

\author{
Nhi Ngoc Yen Huynh ${ }^{1}$, Dai Duc Nguyen ${ }^{1}$, Nguyen Ha Ta ${ }^{1}$, Minh Thien Nguyen, \\ Tai Van Nguyen ${ }^{1}$, Huy Thanh Dang, Tuyen Kim Cat Vo ${ }^{2}$, Ngoan Tran LE ${ }^{3,4}$
}

${ }^{1}$ School of Medicine, International University of Health and Welfare, Japan. ${ }^{2}$ Department of Obstetrics and Gynecology, Graduate School, International University of Health and Welfare, Japan. ${ }^{3}$ Institute of Research and Development, Duy Tan University, Da Nang, Vietnam. ${ }^{4}$ Department of Public Health, School of Medicine, International University of Health and Welfare, Japan.

\begin{abstract}
Aims: This study aimed to describe the characteristics of the workplace-related clusters of COVID-19 and its transmissions into communities in Vietnam. Methods and Material: We accessed the database of COVID-19 by the Ministry of Health, Vietnam. Variables included sources of infection, age, sex, nationality, the dates of onset of symptoms and discharge from hospitals, and ID of each patient tested positive with COVID-19. Information from each patient was linked to the sources of infection to identify workplace-related clusters. Among 314 patients, we excluded 43 cases related to two charter flights, the remaining 271 cases were eligible for the study. Results: The biggest cluster of hospital canteen included 26 workers and their 31 family members or hospital's patients. The second biggest cluster included a pilot of the Vietnam airlines and other 16 patients who have close contact with him at the Bar Buddha at Ho Chi Minh City. A total of 87 patients ( $32.1 \%$ of 271 cases) were related to these workplace-related clusters. The suspected time and the clinical course was significantly longer in the workplace-related clusters than other patients (mean 6.52 vs. 4.05 days, $p=0.0191$ ) and (mean 28.71 vs. 20.52 days, $p=0.0005$ ), respectively. Conclusions: Because COVID-19 infection at workplaces was responsible for nearly one-third of the total patients, there was a novel emerged occupational risk factor at work due to coronavirus infection. Safety at the workplace in preventing COVID-19 transmission is highly needed.
\end{abstract}

Keywords: Workplaces- Coronavirus- COVID-19- Vietnam

Asian Pac Environ Cancer, 3 (1), 27-33

\section{Introduction}

Since December 2019, COVID-19 has caused outbreaks of atypical pneumonia in Wuhan, China, which subsequently became a pandemic all over the world. By May 18, 2020, the disease had spread to 216 countries, areas, and territories, and WHO had recorded 4,628,903 cases of COVID-19 worldwide and attributed 312,009 deaths to the disease [1]. This disease was confirmed to be transmissible from person to person and seemed to cause clusters of disease in healthcare workers (HCWs) [2]. Moreover, the presentation of COVID-19 has ranged from asymptomatic/ mild symptoms to severe illness with prolonged incubation time [3-4-5], which makes the virus
Submission Date: 07/23/2020Ａcceptance Date: 08/01/2020

\footnotetext{
Corresponding Author:

Dr. Ngoan Tran LE

Institute of Research and Development, Duy Tan University, Da Nang, Vietnam. Department of Public Health, School of Medicine, International

University of Health and Welfare, Japan.

Email: letngoan@hmu.edu.vn
}

easily spread in communities.

Vietnam, located in Southeast Asia, sharing the long border with China to the North was supposed to have a high risk of being affected by the pandemic. Besides, with a population of over 97 million in 2020, Vietnam is among the countries with high population density. Given the geographical and demographical features, Vietnam is at a high risk of importing COVID-19 from oversea with the highest number of patients concentrated in Ha Noi and Ho Chi Minh City [6].

Vietnam confirmed its first cases of COVID-19 on 23 January 2020. The first patient was a 66-year-old man from 
Wuhan, China, visiting Vietnam with his wife and met his son in Nha Trang, Vietnam who was also confirmed positive with COVID-19 on the same day. A female hotel receptionist who had contact history with the first patient was diagnosed with COVID-19 on February 1, 2020, and this cluster was determined to be the first work-related cluster in Vietnam [6-7].

Although most of Vietnam is in a tropical climate with high temperatures and humidity clarified to be a disadvantage for the virus's survival [8], the recent increase in using indoor air conditioners in the workplace might create a closed environment with relatively cold temperatures and low humidity that facilitate the viral transmission. For these reasons, common workplace such as factories, schools, hospitals, workshops, offices might have a higher risk of SARS-CoV-2 infection, and once infected, these workers are more likely to develop severe manifestations of disease caused by the virus and spread into their communities. This study aimed to describe the characteristics of the workplace-related clusters of COVID-19 and its transmissions into communities in Vietnam.

\section{Materials and Methods}

\section{Study design and data source}

This study was designed to describe the epidemiological characteristics of 8 workplace-related clusters of COVID-19 in Vietnam using descriptive epidemiological methods and to explore the differences in clinical indicators including incubation time, suspected time, clinical course, and clearance time between the workplace-related and the non-workplace related group. Data regarding patients' age, sex, nationality, the dates of onset of symptoms, and discharge from hospitals were extracted from official information published by the Ministry of Health, Vietnam.

\section{Work-related cluster concept}

A group of patients tested positive with COVID-19 included at least one worker or officer of a recognized institution with formal economical activities was defined as a cluster in the present study. The first case might be an imported case (F0) and the case had a rich contact with F0 case was the local case (F1) and the other local case had close contact with F1 was categorized as an F2. A workplace-associated cluster refers to an aggregation of cases grouped in one particular workplace and time. The number of cases in one cluster is usually suspected to be bigger, even though the expected number may not be known [9]. Tracing and testing information from each patient was linked to sources of agents to identify workplace-related clusters.

\section{Inclusion and exclusion of study participants}

By 16 May 2020, 314 patients tested positive with COVID-19. We excluded 43 cases related to two charter flights. The remaining 271 cases were eligible for the present study. Only cases of infected patients within communities were included in the analysis, charter flight cases were excluded.

\section{The derived indicators}

The derived indicators are calculated as follows: (1) an identified incubation or asymptomatic time was the time from the first exposure day to the date of onset of symptoms and its data available for 77 patients. (2) The suspected time was the time from the date of onset of symptoms to the date confirmed positive with the virus and its data available for 89 patients. (3) A clinical course was the time from the date of onset of symptoms to the discharge date and its data available for 84 patients. (4) A clearance time was the time from the date confirmed positive with COVID-19 to the discharge date and its data available for 260 patients.

\section{Data management and analysis}

Data were reviewed between different sources, cleaned and encoded by Microsoft Excel. STATA 14.0 was used to analyze research data. These indicators were used in the analysis to explore the differences between 2 groups using the T-test. All p-values are two-sided and 0.05 ( $\alpha$ value) was considered the threshold to indicate statistical significance.

\section{Ethical approval}

The research team has used data extracted from the Ministry of Health's COVID-19 information page [6], which provided essential information to help the procedure of preventing the community spread. Name, personal address, date of birth, and other private information of COVID-19 patients were presented neither on the information page nor in the database, therefore ethical guideline is not applied in the present study.

\section{Results}

There were 8 clusters identified as follows, cluster-1 (C1): Patient number 3 had close contact with the imported patient number 1 who was from Wuhan, China, at a hotel where she was working in Nha Trang city. Cluster-2 (C2): Patient number 35 had close contact with the two imported patient number $22 \& 23$ who were from the UK, at an electronic store (her workplace) in Da Nang city. Cluster-3 (C3): Patient number 39 had close contact with the imported patient number 24 who was from the UK and a member of a tour from Hanoi to Ninh Binh city, in which Patient number 39 worked as tour-guide. Cluster-4 (C4): Patients number $46 \& 59$ were the crewmembers of Vietnam Airlines of the Hanoi-London-Hanoi route and they were infected on the airplane during their working services. Cluster-5 (C5): Patient number 91 was a pilot working for Vietnam Airlines of the Hanoi-London-Hanoi route and he was infected on the airplane. Later, the other 16 patients had close contact with him at the Bar Buddha in Ho Chi Minh City. Cluster-6 (C6): The cluster occurred at the workplace of Bach Mai hospital's canteen. A total of 57 patients were infected and related to this cluster. The source of infection was unknown. Cluster-7 (C7): Two physicians were infected from COVID-19 patients at the emergency care unit for COVID-19 of the National Tropical Infectious Disease Hospital. Cluster-8 (C8): 

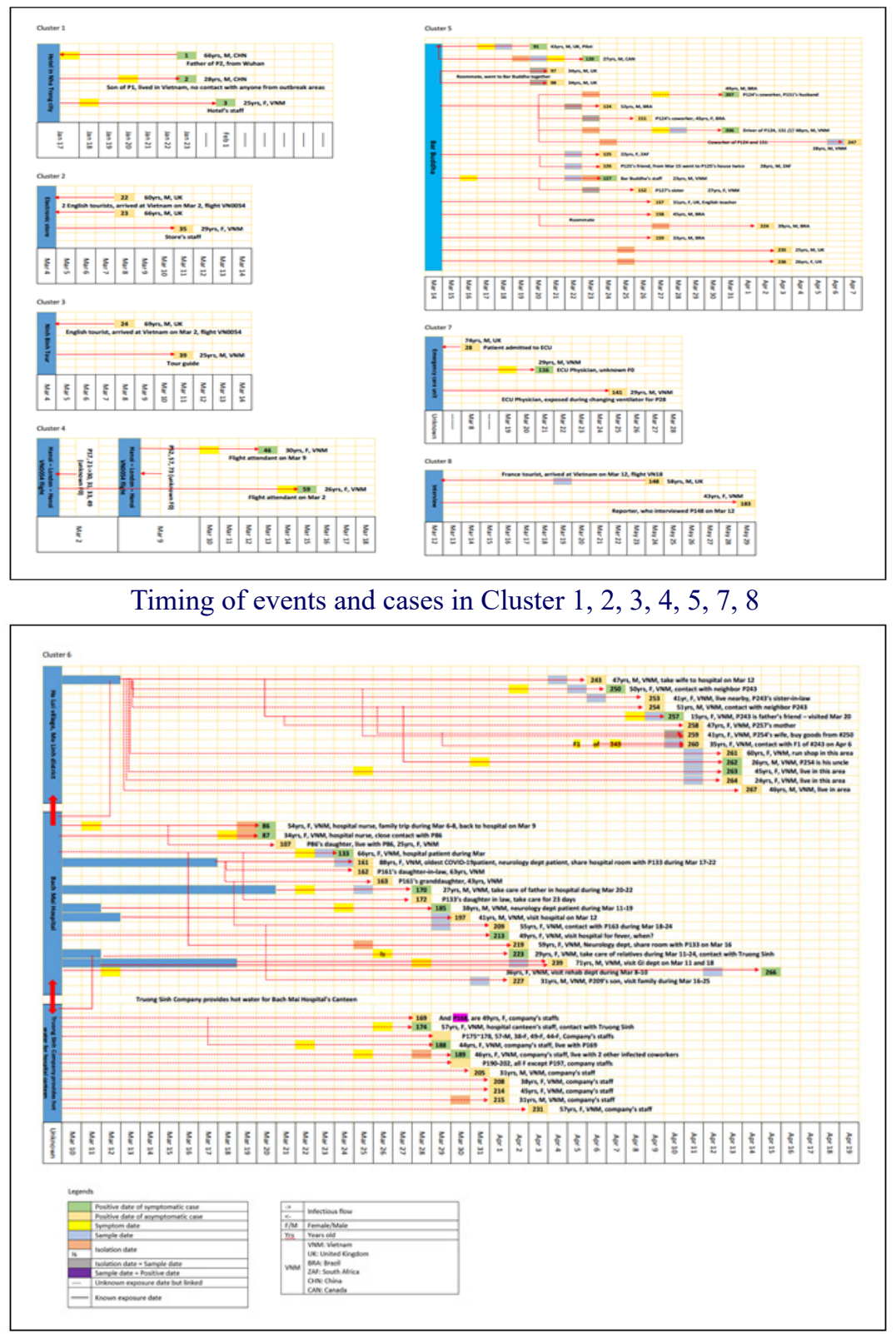

Timing of events and cases in Cluster 6

Figure 1. Timing of Events and Cases in Cluster 1, 2, 3, 4, 5, 7, 8 and 6 in Vietnam

Patient number 183 who is a journalist and had close contact with patient number 148 when he was working (Table 1) (Figure 1).

Table 2 showed the number of patients positive with COVID-19 in Vietnam by May 6, 2020, by age group and gender in the non-work-related and work-related group. In the non-work-related group, the number of patients positive with COVID-19 was concentrated on working ages and decreased consistently with age. There were 25 patients under the age of twenty. The number of patients increased to a peak of 78 patients in the 20-29 age group, followed by a significant drop to 28 patients in the next age group. In the age group of the $40 \mathrm{~s}, 50 \mathrm{~s}$, and $60 \mathrm{~s}$, the number of patients was relatively small, which was 17 , 19 , and 13 , respectively. There were only 4 patients over the age of 70 in the non-work-related group. There was a notable difference in the percentage of male $(36.8 \%)$ and female $(63.2 \%)$ patients in the work-related group, while this gap was insignificant in the non-work-related group.

Table 3 showed a comparison between the nonwork-related and the work-related group, regarding age, incubation period, suspected time, clinical course, and clearance time. Regarding age, the mean age of patients included in the non-work-related group is 32.96 years, compared to 41.98 years of the mean age of the work-related group. The difference is 9.02 years and has statistical significance $(\mathrm{p}=0.0001)$. Moreover, the age of the youngest patient in the non-work-related group is 0.25 years, by contrast, the number is 15 years in the work-related group. These results suggested that the patients in the work-related group tend to be older than the remaining group. The suspected time in the work-related group (6.52 days) is also significantly longer, $\mathrm{p}=0.0191$ than that in the non-work-related group 
Table 1. Eight Clusters of COVID-19 Occurrences in Vietnam

\begin{tabular}{|c|c|c|c|c|c|c|}
\hline \multirow[b]{2}{*}{ Cluster (C) } & \multicolumn{6}{|c|}{ The Date of Cases Tested Positive with COVID-19 } \\
\hline & F0 & $\mathrm{F} 1 \& \mathrm{~F} 2$ & Started date & Finished date & Total cases & Percent \\
\hline $\mathrm{C} 1$ & $\mathrm{P} 1$ & P3 & 23-Jan-20 & $1-\mathrm{Feb}-20$ & 2 & 2.3 \\
\hline \multirow[t]{2}{*}{$\mathrm{C} 2$} & $\mathrm{P} 22$ & P35 & 8-Mar-20 & 11-Mar-20 & 3 & 3.4 \\
\hline & $\mathrm{P} 23$ & & & & & \\
\hline $\mathrm{C} 3$ & $\mathrm{P} 24$ & P39 & 8-Mar-20 & 11-Mar-20 & 2 & 2.3 \\
\hline \multirow[t]{2}{*}{$\mathrm{C} 4$} & unknown & P46 & & & & \\
\hline & & P59 & & 15-Mar-20 & 2 & 2.3 \\
\hline $\mathrm{C} 5$ & P91 & 16 patients & 24-Mar-20 & 7-Apr-20 & 17 & 19.5 \\
\hline C6 & unknown & 57 patients & 20-Mar-20 & $15-A p r-20$ & 57 & 65.5 \\
\hline $\mathrm{C} 7$ & unknown & 2 Physicians & 20-Mar-20 & 25-Mar-20 & 2 & 2.3 \\
\hline $\mathrm{C} 8$ & P148 & P183 & 26-Mar-20 & 29-Mar-20 & 2 & 2.3 \\
\hline Total & & & & & 87 & 100 \\
\hline
\end{tabular}

Table 2. Study Participants by Age Group and Sex

\begin{tabular}{|c|c|c|c|c|c|c|}
\hline \multirow{2}{*}{$\begin{array}{l}\text { Variable } \\
\text { Age group }\end{array}$} & \multicolumn{2}{|c|}{ Non-work-related COVID-19 } & \multicolumn{2}{|c|}{ Work-related COVID-19 } & \multicolumn{2}{|c|}{ Total } \\
\hline & Patients & Percent & Patients & Percent & Patients & Percent \\
\hline $0-19$ & 25 & 13.6 & 1 & 1.1 & 26 & 9.6 \\
\hline $20-29$ & 78 & 42.4 & 22 & 25.3 & 100 & 36.9 \\
\hline $30-39$ & 28 & 15.2 & 15 & 17.2 & 43 & 15.9 \\
\hline $40-49$ & 17 & 9.2 & 26 & 29.9 & 43 & 15.9 \\
\hline $50-59$ & 19 & 10.3 & 13 & 14.9 & 32 & 11.8 \\
\hline $60-69$ & 13 & 7.1 & 8 & 9.2 & 21 & 7.7 \\
\hline $70+$ & 4 & 2.2 & 2 & 2.3 & 6 & 2.2 \\
\hline Total & 184 & 100 & 87 & 100 & 271 & 100 \\
\hline \multicolumn{7}{|l|}{ Sex } \\
\hline men & 93 & 50.5 & 32 & 36.8 & 125 & 46.1 \\
\hline women & 91 & 49.5 & 55 & 63.2 & 146 & 53.9 \\
\hline Total & 184 & 100 & 87 & 100 & 271 & 100 \\
\hline
\end{tabular}

(4.05 days). Similarly, the difference in mean clinical courses between the two groups (20.52 days versus 28.71 days) is 8.19 days, which is also statistically significant $(p=0.0005)$. Finally, clearance time follows a similar trend: the clearance time in the non-work-related and the work-related group is 18.35 days and 21.56 days, respectively. The difference is statistically significant: 3.21 days $(p=0.0097)$. In contrast, considering the mean incubation time, there was no significant difference between the non-work-related and the work-related group, the mean incubation period is 7.65 and 8.04 , respectively, $\mathrm{p}=0.3952$.

\section{Discussion}

We observed the differences with statistical significance regarding suspected time, mean clinical course, and clearance time between the non-work-related and the work-related clusters. Longer clinical course and clearance time suggested the complication of Covid-19 disease courses in the workplace-related cluster compared to non-workplace-related patients, which underscore the importance of proactive steps to appropriately prevent and control the disease spreading. Lessons learned from this study can provide valuable guidance for containing

Table 3. Early Outcome of COVID-19 Management and Treatment

\begin{tabular}{lcccccccccccc}
\hline & \multicolumn{4}{c}{ Non-work-related COVID-19 } & \multicolumn{4}{c}{ Work-related COVID-19 } & \multicolumn{3}{c}{ Difference } & T-test \\
Variable & $\mathrm{n}$ & Mean (1) & S.D. & Min & Max & $\mathrm{n}$ & Mean (2) & S.D. & Min & Max & $(2)-(1)$ & $\mathrm{p}$ \\
\hline Age & 184 & 32.96 & 15.91 & 0.25 & 74 & 87 & 41.98 & 14.14 & 15 & 88 & 9.02 & 0.0001 \\
Incubation & 52 & 7.65 & 6.05 & 0 & 25 & 25 & 8.04 & 5.91 & 1 & 24 & 0.39 & 0.3952 \\
Suspected time & 62 & 4.05 & 4.15 & 0 & 28 & 27 & 6.52 & 6.8 & 1 & 33 & 2.47 & 0.0191 \\
Clinical course & 60 & 20.52 & 9.49 & 6 & 64 & 24 & 28.71 & 11.19 & 16 & 51 & 8.19 & 0.0005 \\
Clearance time & 178 & 18.35 & 9.74 & 1 & 61 & 82 & 21.56 & 11.21 & 3 & 50 & 3.21 & 0.0097 \\
\hline
\end{tabular}


workplace-related COVID-19 spread in Vietnam and other countries. Because COVID-19 infection at workplaces was responsible for nearly one-third of the total patients reported in Vietnam by May 6, 2020, there was a novel emerging occupational risk factor at work due to coronavirus infection.

In general, the number of patients tested positive with COVID-19 was highest in the age group of the 20 s in the non-workplace-related group, and by contrast, this number spreader more sporadically in the workplace-related group. Also, the mean age of the work-related group is higher. These can be explained by the fact that individuals belonging to the former group are mainly international students and foreign workers who were early detected and isolated at airports [10]. Meanwhile, work-related clusters of unknown sources tend to involve people from a wider range of age, including patients' family members and patients' customers. In the case of nosocomial infection that occurred in Bach Mai hospital, patients confirmed positive with COVID-19 included in-patients and out-patients of the hospital, family members taking care of those patients, healthcare workers, and their neighbors [10].

The number of cases that are older than 40 in both work-related and non-work-related groups consisted of only around one-tenth of the total number of patients and slowly decreased by age. This suggested that, in Vietnam, COVID-19 is mainly spread among young people. A systematic review study had shown asymptomatic patients can spread the virus [11], therefore, we speculated that active roles of young patients in work-place settings and family settings can be the cause of transmission of COVID-19 in communities. For that reason, we suggested that enhancing awareness of young people about COVID-19 is crucial in controlling this disease.

The mean clinical course of the work-related group is longer (8.19 days) than that of the non-work-related group. Since the clinical course equals the sum of suspected time and clearance time, the longer clinical course can be attributed to either longer suspected time, clearance time, or both. According to our study, both suspected time and clearance time in the work-related group are longer than those in the non-work-related group. First, we propose an explanation for the long-suspected time in the work-related group. As mentioned earlier, the non-workrelated group includes many international workers and students imported into Vietnam, who were all mandatorily immediately quarantined and tested, hence the time delay between their onset of symptoms and confirmation of being positive (i.e suspected time) is shorter. In contrast, the suspected time in the work-related group is longer (2.47 days), probably explained by the facts that patients in the work-related group tended to delay seeking medical attention and testing for some reasons (no mandatory testing, neglecting the importance of early diagnosis, being overwhelmed by work, positive diagnosis may interfere with the current job). Second, we explain why clearance time in the work-related group is also longer (3.21 days) than the remaining group. Longer suspected time also helps explain longer clearance time: the delay in seeking early medical attention and testing leads to a more severe trajectory and hence delayed healing. Besides longer suspected time, we speculate that other explanations exist. First, the mean age of the work-related group was higher than the mean age of the non-work-related group, and older people are more likely to become severe cases and have delayed recovery [12-13-14-15]. Second, there are some unknown risk factors related to the workplace that cause work-related patients to suffer from more severe outcomes and delayed healing: close, multiple and prolonged contacts with carriers, as normally happening in the workplace, resulting in inhaling more viral particles which leads to more severe disease. Although this assumption has not been studied in COVID-19 due to its severity, it was studied in the case of Influenza, in which volunteers exposed to more viral particles experienced more severe symptoms [16].

There was no statistically significant difference between the non-work-related and the work-related group, regarding the mean incubation.

Workers tend to delay seeking medical evaluation even when they have symptoms, which increases the chance of transmission in the workplace and can cause serious outbreaks. Therefore, having workers report daily health conditions, including body temperature and respiratory symptoms, is crucial. Since COVID-19 symptoms can be mild [3-4], workers tend to go to work despite having symptoms, or "presenteeism". Measures to enhance awareness of workers about COVID-19 like encouraging individual responsibility to stay at home if unwell, and to actively seek medical help is critical to prevent workplace outbreak. Intensive PCR testing and quarantine for high-risk individuals and cohorts at their workplaces and communities are also important to early detect and prevent the spread of COVID-19.

In the non-healthcare-related group, universal measures must be implemented cautiously to protect them from COVID-19. These measures include hand washing, wearing masks, social distancing, disinfection of the environment, and enhancing awareness of workers. According to $\mathrm{WHO}$, proper and frequent hand washing is one of the most crucial measures to prevent COVID-19 [17]. Although personal protective equipment (PPE) is not recommended for workers in non-healthcare settings, surgical masks should be worn by people with respiratory symptoms to avoid the spreading of the droplets [18]. Moreover, given the possibility of close human to human contact in workplace settings, the physical distance must be kept between worker-customer and worker-worker. In an experiment examining the stability of SARS-CoV-2 applied on different surfaces, viable viruses were detected up to 72 hours after application to stainless steel and plastic surfaces [19]. Therefore, routine disinfection of frequently touched surfaces in the workplace is also recommended to prevent COVID-19 [20].

For the healthcare-related group, a recent article stated the reasons for nosocomial infection in China as inadequate personal protection, long time exposure to a large number of infected patients, shortage of PPE, and lack of training for infection prevention and control [21]. 
In terms of infection prevention and control measures, several reports indicated that there were no secondary infections in healthcare workers exposed to infected patients while using only contact and droplet precautions, in the absence of airborne precautions [22-23]. Therefore, applying standard precautions for all patients, and additional droplet and contact precautions for patients with COVID-19 is crucial to limit the spread of COVID-19 in the hospital and protect the HCWs and patients [18]. Second, as a shortage of PPE is an ongoing difficult problem, support from society and manufacturers is essential to ensure sufficient supply for the HCWs. Lastly, raising the awareness of HCWs about infection prevention and control, especially the importance and indication of PPE in each situation plays an important role in COVID-19 control. Studies concluded that the use of PPE and infection control training is associated with a decreased risk of coronavirus infection [24].

The Bach Mai hospital and the government have conducted comprehensive measures, including mass screening of staff, patients, and caregivers; sufficient PPE supply and proper wearing practice; hospital blockade and suspected cases isolation; disinfection of the entire hospital; announcing hotlines to receive information of possibly related people, etc. As a result, the situation in Bach Mai hospital has been contained well within less than one month, where only 2 among 57 cases are HCWs. This result is consistent with the study emphasizing the role of comprehensive surveillance strategy, outbreak management, and individual responsibility to achieve success in early detection of COVID-19 clusters among HCWs [25].

Vietnam has been successful in containing COVID-19 with only 314 confirmed cases (or 2.9 cases per million people), despite having a $1400 \mathrm{~km}$ border with China and a large population of 97 million people [6]. This success can be attributed to the early and decisive action of Vietnam's Government. Vietnam Ministry of Health together with other relevant ministries implemented unprecedented Covid-19 control measures, including strictly controlling all points of entry, halting all flights from high-risk areas, conducting immediate suspected cases quarantine, and comprehensive contact tracing [26-27-28].

Our study limitations include the small sample size available for data analysis. Moreover, we were not able to evaluate patients' basic health conditions in each compared group, one factor that might affect the result of our study. We found differences with statistical significance regarding mean age, gender, mean clinical course, and clearance time between the non-work-related and the work-related group. Although there is interest in finding the underlying reasons, we are unable to fully address this point in our study, and further studies are warranted to elucidate the main findings in our study.

\section{Acknowledgments}

We thank Physicians and Nurses, Officers of the Ministry of Health for their hard work of testing, tracing, isolating, and treating activities in controlling the ongoing
Coronavirus pandemic in Vietnam.

Source(s) of support

There was no support.

Conflicting Interest (If present, give more details):

There are no conflicts of interest to disclose.

\section{References}

1. World Health Organization. Coronavirus disease (COVID-19) outbreak situation in2020.[Online]. Available at: https://www. who.int/emergencies/diseases/novel-coronavirus-2019.

2. Wei X, Wang X, Zhang J, Yang W, Ma W, Yang B, Jiang N, Gao Z, Shi H, Zhou Q. A cluster of health care workers with COVID-19 pneumonia caused by SARS-CoV-2. Journal of Microbiology, Immunology and Infection. 2020 04;. https:// doi.org/10.1016/j.jmii.2020.04.013

3. Chan JF, Yuan S, Kok K, To KK, Chu H, Yang J, Xing F, Liu J, Yip CC, Poon RW, Tsoi H, Lo SK, Chan K, Poon VK, Chan W, Ip JD, Cai J, Cheng VC, Chen H, Hui CK, Yuen K. A familial cluster of pneumonia associated with the 2019 novel coronavirus indicating person-to-person transmission: a study of a family cluster. The Lancet. 2020 02;395(10223):514-523. https://doi.org/10.1016/s01406736(20)30154-9

4. Zhou F, Yu T, Du R, Fan G, Liu Y, Liu Z, Xiang J, Wang Y, Song B, Gu X, Guan L, Wei Y, Li H, Wu X, Xu J, Tu S, Zhang Y, Chen H, Cao B. Clinical course and risk factors for mortality of adult inpatients with COVID-19 in Wuhan, China: a retrospective cohort study. The Lancet. 2020 03;395(10229):1054-1062. https://doi.org/10.1016/s01406736(20)30566-3

5. Lauer SA, Grantz KH, Bi Q, Jones FK, Zheng Q, Meredith HR, Azman AS, Reich NG, Lessler J. The Incubation Period of Coronavirus Disease 2019 (COVID-19) From Publicly Reported Confirmed Cases: Estimation and Application. Annals of Internal Medicine. 202005 05;172(9):577-582. https://doi.org/10.7326/m20-0504

6. Ministry of Health of Vietnam. Information Page about COVID-19 Respiratory Disease Outbreak 2020. [Online]. Available at: https://ncov.moh.gov.vn/..

7. Phan LT, Nguyen TV, Luong QC, Nguyen TV, Nguyen HT, Le HQ, Nguyen TT, Cao TM, Pham QD. Importation and Human-to-Human Transmission of a Novel Coronavirus in Vietnam. New England Journal of Medicine. 202002 27;382(9):872-874. https://doi.org/10.1056/nejmc2001272

8. Wolkoff P. Indoor air humidity, air quality, and health - An overview. International Journal of Hygiene and Environmental Health. 2018 04;221(3):376-390. https://doi. org/10.1016/j.ijheh.2018.01.015

9. CDC. Principles of Epidemiology in Public Health Practice. Lesson 1: Introduction to Epidemiology. Section 11: Epidemic Disease Occurrence. [Online]. Available at: https:// www.cdc.gov/csels/dsepd/ss1978/lesson1/section11.html..

10. Ministry of Health of Vietnam. Statistics of COVID-19 in Vietnam 2020. [Online]. Available at: https://ncov.vncdc. gov.vn/..

11. Gao Z, Xu Y, Sun C, Wang X, Guo Y, Qiu S, Ma K. A systematic review of asymptomatic infections with COVID-19. Journal of Microbiology, Immunology and Infection. 2020 05; https://doi.org/10.1016/j.jmii.2020.05.001

12. Verity R, Okell LC, Dorigatti I, Winskill P, Whittaker C, Imai N, Cuomo-Dannenburg G, Thompson H, Walker PGT, Fu H, Dighe A, Griffin JT, Baguelin M, Bhatia S, Boonyasiri 
A, Cori A, Cucunubá Z, FitzJohn R, Gaythorpe K, Green W, Hamlet A, Hinsley W, Laydon D, Nedjati-Gilani G, Riley S, van Elsland S, Volz E, Wang H, Wang Y, Xi X, Donnelly CA, Ghani AC, Ferguson NM. Estimates of the severity of coronavirus disease 2019: a model-based analysis. The Lancet Infectious Diseases. 2020 06;20(6):669-677. https:// doi.org/10.1016/s1473-3099(20)30243-7

13. Chen N, Zhou M, Dong X, Qu J, Gong F, Han Y, Qiu Y, Wang J, Liu Y, Wei Y, Xia J, Yu T, Zhang X, Zhang L. Epidemiological and clinical characteristics of 99 cases of 2019 novel coronavirus pneumonia in Wuhan, China: a descriptive study. The Lancet. 2020 02;395(10223):507-513. https://doi.org/10.1016/s0140-6736(20)30211-7

14. Wang D, Hu B, Hu C, Zhu F, Liu X, Zhang J, Wang B, Xiang H, Cheng Z, Xiong Y, Zhao Y, Li Y, Wang X, Peng Z. Clinical Characteristics of 138 Hospitalized Patients With 2019 Novel Coronavirus-Infected Pneumonia in Wuhan, China. JAMA. 202003 17;323(11):1061. https://doi.org/10.1001/ jama.2020.1585

15. McGonagle D, O'Donnell JS, Sharif K, Emery P, Bridgewood C. Immune mechanisms of pulmonary intravascular coagulopathy in COVID-19 pneumonia. The Lancet Rheumatology. 2020 07;2(7):e437-e445. https://doi. org/10.1016/s2665-9913(20)30121-1

16. Memoli MJ, Czajkowski L, Reed S, Athota R, Bristol T, Proudfoot K, Fargis S, Stein M, Dunfee RL, Shaw PA, Davey RT, Taubenberger JK. Validation of the Wild-type Influenza A Human Challenge Model H1N1pdMIST: An A(H1N1) pdm09 Dose-Finding Investigational New Drug Study. Clinical Infectious Diseases. 2014 Nov 20;60(5):693-702. https://doi.org/10.1093/cid/ciu924

17. World Health Organization. Water, sanitation, hygiene, and waste management for the COVID-19 virus: interim guidance 2020. [Online]. Available at: https://www.who. int/publications-detail/water-sanitation-hygiene-and-wastemanagement-for-the-covid-19-virus-interim-guidance..

18. World Health Organization. Rational use of personal protective equipment for coronavirus disease (COVID-19) and considerations during severe shortages in 2020. [Online]. Available at: https://www.who.int/publicationsdetail/rational-use-of-personal-protective-equipment-forcoronavirus-disease-(COVID-19)-and-considerationsduring-severe-shortages..

19. van Doremalen N, Bushmaker T, Morris DH, Holbrook MG, Gamble A, Williamson BN, Tamin A, Harcourt JL, Thornburg NJ, Gerber SI, Lloyd-Smith JO, de Wit E, Munster VJ. Aerosol and Surface Stability of SARS-CoV-2 as Compared with SARS-CoV-1. New England Journal of Medicine. 202004 16;382(16):1564-1567. https://doi. org/10.1056/nejmc2004973

20. World Health Organization. Advice on the use of masks in the context of COVID-192020. [Online]. Available at: https://www.who.int/publications-detail/advice-on-theuse-of-masks-in-the-community-during-home-care-and-inhealthcare-settings-in-the-context-of-the-novel-coronavirusoutbreak.

21. Wang J, Zhou M, Liu F. Reasons for healthcare workers becoming infected with novel coronavirus disease 2019 (COVID-19) in China. Journal of Hospital Infection. 2020 05;105(1):100-101. https://doi.org/10.1016/j. jhin.2020.03.002

22. Ng K, Poon BH, Kiat Puar TH, Shan Quah JL, Loh WJ, Wong YJ, Tan TY, Raghuram J. COVID-19 and the Risk to Health Care Workers: A Case Report. Annals of Internal Medicine. 202006 02;172(11):766-767. https://doi. org/10.7326/120-0175

23. Wong S, Kwong R, Wu T, Chan J, Chu M, Lee S, Wong H,
Lung D. Risk of nosocomial transmission of coronavirus disease 2019: an experience in a general ward setting in Hong Kong. Journal of Hospital Infection. 2020 06;105(2):119127. https://doi.org/10.1016/j.jhin.2020.03.036

24. Chou R, Dana T, Buckley DI, Selph S, Fu R, Totten AM. Epidemiology of and Risk Factors for Coronavirus Infection in Health Care Workers. Annals of Internal Medicine. 2020 07 21;173(2):120-136. https://doi.org/10.7326/m20-1632

25. Wee LE, Sim XYJ, Conceicao EP, Aung MK, Goh JQ, Yeo DWT, Gan WH, Chua YY, Wijaya L, Tan TT, Tan BH, Ling ML, Venkatachalam I. Containment of COVID-19 cases among healthcare workers: The role of surveillance, early detection, and outbreak management. Infection Control \& Hospital Epidemiology. 202005 11;41(7):765-771. https:// doi.org/10.1017/ice.2020.219

26. Ministry of Health of Vietnam. Situation and measures to prevent and combat acute pneumonia caused by new coronavirus strain in Wuhan city, Hubei province, China. General Department of preventive medicine, 2020. [Online]. Available at: http://vncdc.gov.vn/vi/tin-tuc-su-kien/8556/ tinh-hinhva-cac-bien-phap-phong-chong-dich-benh-viemphoi-cap-do-chung-coronavirus-moi-taithanh-pho-vu-hantinh-ho-bac-Trung-Quoc.

27. Vietnam Prime Minister. Directive No. 05/CT-TTG dated January 28, 2020, of the Prime Minister on the prevention and control of acute respiratory infections caused by new strains of novel Coronavirus. Vietnam's Government, 2020..

28. Ministry of Health of Vietnam. Mandatory medical data collection for every passengers' entries to Vietnam. Ministry of Health Portal, 2020. [Online]. Available at: https:// moh.gov.vn/hoat-dong-cua-dia-phuong/-/asset_publisher/ gHbla8vOQDuS/content/khai-bao-y-te-bat-buoc-moi-hanhkhach-nhap-canh-vao-Viet-Nam.

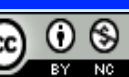

This work is licensed under a Creative Commons AttributionNon Commercial 4.0 International License. 